\title{
Silicon molecules in space and in the laboratory
}

\author{
M. C. McCARTHY, C. A. GOTTLIEB and P. THADDEUS* \\ Harvard-Smithsonian Center for Astrophysics, Cambridge, MA 02138 and Division \\ of Engineering and Applied Sciences, Harvard University, Cambridge, MA 02138, USA
}

(Received 12 August 2002; accepted 22 August 2002)

\begin{abstract}
Despite the highly non-volatile nature of $\mathrm{Si}$, silicon bearing molecules are a significant trace constituent of the gas associated with late-type stars and star forming regions, representing nearly $10 \%$ of the molecular species that have been identified in space. The early astronomical detection of $\mathrm{SiO}$ and its remarkable maser emission stimulated laboratory studies of other small silicon species that were crucial to the astronomical identifications. Although the pace of this laboratory work until recently has been slow, the application of Fourier transform microwave spectroscopy to supersonic molecular beams has overcome many experimental difficulties, yielding the detection of more than 20 new silicon chains and rings of astronomical interest during the past three years. Three of these, the rhomboidal ring $\mathrm{SiC}_{3}$ and the isovalent linear molecules $\mathrm{SiCN}$ and $\mathrm{SiNC}$, have now been found in space, and with dedicated searches other recently discovered silicon molecules may soon be found. A number of exotic silicon molecules of astronomical and chemical interest should be amenable to laboratory detection with present techniques.
\end{abstract}

\section{Introduction}

Silicon on Earth is considered a highly non-volatile element, and there are essentially no gas-phase silicon containing molecules in the terrestrial atmosphere, which (excepting argon) is composed almost entirely of elements from the first row of the Periodic Table. In striking contrast, however, $\mathrm{Si}$ in the interstellar gas is only slightly more depleted than $\mathrm{C}, \mathrm{N}$ and $\mathrm{O}$ [1] and, in some circumstellar shells (the gas streaming outward from evolved stars), a surprisingly rich silicon chemistry has been discovered, even in regions where the temperature is fairly low. Of the 130 or so gas-phase astronomical molecules, 10 contain silicon (see figure 1), and it is likely that with better spectroscopic data from the laboratory, many more may be found.

The radio astronomical observations of $\mathrm{SiO}$ in space in the early 1970s [2] and the discovery of its remarkable maser emission in vibrationally excited states [3-5] provided an important impetus to the laboratory rotational spectroscopy of silicon molecules. Nevertheless the laboratory work proceeded slowly in the succeeding twenty years, owing to the difficulty of producing these highly refractory molecules in the gas phase in adequate concentrations. In the early laboratory experiments, $\mathrm{SiO}$ [6-8] and SiS [9] were produced by resistively heating refractory silicon compounds to high temperatures. Although this approach yielded the identification of

* Author for correspondence. e-mail: pthaddeus@cfa. harvard. edu
$\mathrm{SiO}$ and $\mathrm{SiS}$ in space, it led to the detection of only a few other silicon molecules in the laboratory, none of which has yet been found in space. While attempting to improve the spectroscopic constants of SiCC, derived at that time mainly on the basis of astronomical data, we discovered that small reactive silicon bearing molecules such as $\mathrm{SiC}, \mathrm{SiC}_{2}$, and $\mathrm{SiO}$ could be produced efficiently in low pressure discharges through mixtures of silane $\left(\mathrm{SiH}_{4}\right)$ and stable gases such as $\mathrm{CO}$ and $\mathrm{C}_{2} \mathrm{H}_{2}$. In the past few years this production method has been adapted to a pulsed supersonic discharge nozzle, resulting in the detection of many new silicon molecules of astronomical interest in this laboratory by means of Fourier transform microwave (FTM) spectroscopy.

Following a brief discussion of the early astronomical discoveries of silicon molecules and of the supporting laboratory spectroscopy, we review the recent laboratory and astronomical detections of three new silicon molecules, and discuss the prospect that other newly found silicon molecules may be found in space with sensitive radio telescopes. We end with a discussion of other silicon molecules of astronomical and chemical interest that may be amenable to laboratory detection.

\section{1. $\mathrm{SiO}$}

Silicon monoxide, $\mathrm{SiO}$, was discovered in space from observation of rotational lines in its ground vibrational state in 1971 [2], but much stronger lines from excited vibrational states were observed accidently three years later [3], and soon shown to be the result of population 
$\mathrm{SiO}$

$\mathrm{SiC}$

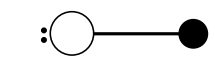

$\mathrm{SiN}$

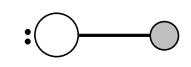

Sis

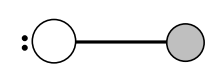

$\mathrm{SiCN}$

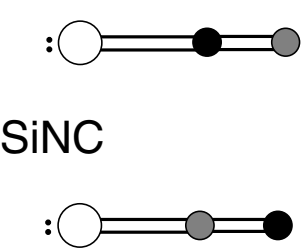

Figure 1. Astronomical silicon-bearing diatomic and polyatomic molecules, all but $\mathrm{SiH}_{4}$ discovered with radio telescopes. $\mathrm{SiH}_{2}$ has been reported also by Turner [59], but because of its small dipole moment $(<0.1 \mathrm{D})$ and the lack of precise laboratory rest frequencies, this detection is tentative. Large open circles denote silicon atoms, large filled circles carbon atoms, large shaded circles hetero atoms $(\mathrm{N}, \mathrm{O}$ or $\mathrm{S})$, and small filled circles hydrogen atoms.

inversion and maser amplification [10]. SiO masers are now among the best studied and understood astronomical masers [11], and $\mathrm{SiO}$ is unique among astronomical diatomic molecules: vibrationally excited maser emission in CO, CS, and SiS has been the subject of extensive, but unsuccessful searches. Radio interferometric maps of $\mathrm{SiO}$ masers have been used widely to investigate at high angular resolution the structure and dynamics of late-type stars and star forming regions. In its ground vibrational state, $\mathrm{SiO}$ is one of the most important molecular species for determining the $\mathrm{C} / \mathrm{O}$ ratio in circumstellar envelopes of late-type stars because, unlike many molecular species, it is readily observed towards both oxygen-rich and carbon-rich stars [12].

\section{2. $\mathrm{SiS}$}

The abundance of silicon monosulphide, SiS, is comparable with or greater than that of $\mathrm{SiO}$ in carbon-rich stars, a remarkable result for a molecule with two second row elements. SiS was first detected towards the carbon-rich star IRC +10216 a few years after $\mathrm{SiO}$ was detected [13], but it has now been observed towards several oxygen-rich stars [12]. Its lines are so intense towards IRC +10216 that SiS has now been observed in the three lowest vibrationally excited states of the main isotopic species, and in the ground vibrational state [14] of six rare isotopic species, including the doubly substituted species ${ }^{29} \mathrm{Si}^{34} \mathrm{~S}$ and ${ }^{30} \mathrm{Si}^{34} \mathrm{~S}$. Millimetre-wave interferometric measurements in IRC+ 10216 show that $\mathrm{SiS}$ is localized near the central star rather than in the outer circumstellar shell [15]. Owing to its high abundance and chemical reactivity, $\mathrm{SiS}$ is thought to be the progenitor of silicon carbides such as $\mathrm{SiC}, \mathrm{SiC}_{2}$, and $\mathrm{SiC}_{4}$, which are thought to be synthesized in the outer circumstellar envelope of carbon-rich stars by the reactions of the abundant positive ions $\mathrm{C}_{2} \mathrm{H}_{2}^{+}, \mathrm{C}_{2} \mathrm{H}_{3}^{+}$, and $\mathrm{C}^{+}$with SiS [16].

\section{3. $\mathrm{SiC}$}

Silicon carbide, $\mathrm{SiC}$, has been found only in the circumstellar shell of the well known evolved carbon star IRC +10216 . Because $\mathrm{SiC}$ is so refractory, and condenses out of the vapour only at high temperatures, generally it was assumed that if it could be found in space it would be close to the surface of a star, and would be of small angular extent [17]. Instead, SiC was found at a considerable distance from the central star in IRC+10216 in a region where the temperature is fairly low [18]. When $\mathrm{SiC}$ was first discovered, Glassgold [16] correctly predicted the abundance of $\mathrm{SiC}$ to be less than that of $\mathrm{SiCC}$ and $\mathrm{SiS}$ and that $\mathrm{SiC}$ would be observed in the outer circumstellar shell because it is the daughter product of a series of reactions starting with SiS and other simple silicon molecules.

Gaseous $\mathrm{SiC}$ eluded high resolution spectroscopic detection for many decades before Bernath et al. [19] observed a transition between two excited electronic states in the near IR. Rotational transitions in the ground electronic state were detected shortly afterwards at millimetre wavelengths in the laboratory and in space [18]. Determination of the ground state constants by Cernicharo et al. [18] allowed Brazier et al. [20] to assign an electronic transition between the ground state and the lowest excited $A^{3} \Sigma^{-}$state. Subsequently, a second transition between the ground state and a higher lying excited electronic state $C^{3} \Pi$ was observed in the laboratory by laser induced fluorescence (LIF) near $438 \mathrm{~nm}[21,22]$. To date, $\mathrm{Si}^{13} \mathrm{C}$ and vibrationally excited $\mathrm{SiC}$ have not been observed in space although they have been measured in the laboratory [23].

\section{4. $\mathrm{SiC}_{2}$}

Silicon dicarbide, $\mathrm{SiC}_{2}$, the three-membered ionic ring represented as $\mathrm{Si}^{+}(\mathrm{C}-\mathrm{C})^{-}$, has a very long history. It was observed first in 1926 by Merrill and Sanford [24] towards carbon-rich stars by its strong blue-green bands 
near $500 \mathrm{~nm}$. Thirty years later $\mathrm{SiC}_{2}$ was observed in the laboratory and was shown to be the carrier of these well known optical bands [25]; however, it was mistakenly thought to be a linear molecule (i.e., $\mathrm{Si}-\mathrm{C}-\mathrm{C}$ ). It was not until 1984 that an elegant two-photon ionization experiment established the ground state geometry: a three-membered ring with $\mathrm{C}_{2 \mathrm{v}}$ symmetry [26]. Approximate rotational constants derived by Michalopoulos et al. [26] allowed Thaddeus et al. [27] to assign nine previously unidentified lines in space to $\mathrm{SiC}_{2}$, to greatly improve the accuracy of the rotational constants, and to determine the leading centrifugal distortion constants. The pure rotational spectrum was measured a few years later in the laboratory by Gottlieb et al. [28] who found indirect evidence for a non-rigid structure. Since then $\mathrm{SiC}_{2}$ has been investigated in a number of laboratories, and has been the subject of several quantum theoretical calculations which have shown that there is large amplitude motion in the low lying $\nu_{3}$ vibrational mode (for a summary see [29]). Many rotational lines of $\mathrm{SiC}_{2}$, including those of the singly substituted isotopic species, have now been observed in IRC +10216 . In addition, rotational lines in the first vibrational state of the $\nu_{3}$ mode have also been observed in this circumstellar shell [14].

\section{5. $\mathrm{SiC}_{4}$}

The linear chain $\mathrm{SiC}_{4}$ was found accidently in IRC +10216 , and its presence there was confirmed by subsequent laboratory detection in the same discharge source in which $\mathrm{SiC}$ and SiCC are observed [30]. Observations of $\mathrm{SiC}_{4}$ at millimetre wavelengths in space and in the laboratory are sparse, owing to the large rotational partition function and the fairly low abundance with respect to other astronomical silicon carbides. In IRC +10216 , the abundance of $\mathrm{SiC}_{4}$ is ten times less than that of $\mathrm{SiC}$ and thirty times less than that of $\mathrm{SiC}_{2}$ [30].

\section{Laboratory and astronomical discovery of new silicon bearing molecules}

Despite advances that had been made in the laboratory detection of reactive silicon molecules of astronomical interest in this laboratory and elsewhere, less than ten such species had been studied by rotational spectroscopy prior to the mid 1990s. Detection of large silicon molecules is an experimental challenge because in addition to their high refractivity, the rotational partition function of these molecules becomes increasing unfavourable in a large discharge cell (even when the walls are cooled with liquid nitrogen to about $100 \mathrm{~K}$ ), because of dilution over many thermally populated levels. Another consideration in our production scheme is safety, since a great deal of care must be exercised in the handling and disposal of unreacted silane gas and its discharge products. Silane is spontaneously flammable in air at concentrations of $5 \%$ or more and, unfortunately, high flows with little dilution were required to produce the strongest signals of $\mathrm{SiC}$ and $\mathrm{SiC}_{2}$ with our free-space millimetre-wave absorption spectrometer.

The application of FTM spectroscopy to supersonic molecular beams has largely overcome these difficulties, yielding here during the past three years the detection of more than two dozen silicon molecules of astronomical interest (figure 2). This technique has proved particularly effective because many types of reactive molecules can be produced efficiently in an electrical discharge source and their rotational spectra are simplified greatly at the low rotational temperature of a few degrees Kelvin that is achieved readily in a supersonic expansion. In addition, stringent safety precautions are not needed, because the flow of silane is about 500 times less than in our millimetre-wave spectrometer, and the silane is heavily diluted in a buffer gas. Rotational lines of many of the new silicon species shown in figure 2 may now be observed with a signal to noise approaching 100 in one minute of integration, sufficient to detect rare isotopic species in natural abundance. Molecular structures have been determined for about one-quarter of the new molecules by isotopic substitution, simply by measuring the rare isotopic species in natural abundance.

In our spectrometer, reactive silicon molecules are created by a small low-current cw discharge in the throat of the expansion nozzle (figure 3) as a gas pulse consisting of a small amount of silane and an organic vapour heavily diluted ( $99 \%$ or more) in an inert gas ( $\mathrm{Ar}$ or $\mathrm{Ne}$ ) expands from a commercial pulsed valve. Free expansion from the nozzle forms in a short distance an approximately mach 2 supersonic beam with a rotational temperature approaching $1 \mathrm{~K}$. As the beam passes through a large high- $Q$ Fabry-Perot microwave cavity, a rotational transition resonant with one of the modes of the cavity is excited coherently by a short pulse of resonant microwave radiation; the radiation almost immediately decays away, and line radiation by the coherently rotating molecules is then detected by a sensitive microwave receiver. The Fourier transform of this free induction decay yields the power spectrum. For an axial beam (the configuration adopted for nearly all our experiments because it gives the best signal-to-noise and resolution) the line shape is double peaked, owing to the two Doppler components that result from decomposition of the confocal mode of the Fabry-Perot cavity into two travelling waves, one parallel to the supersonic beam and the other antiparallel. In practice, this peculiar lineshape is usually only a minor inconvenience, and the effective spectral resolution of the spectrometer is 


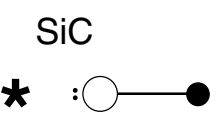

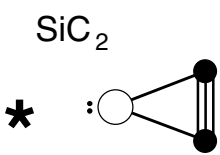

$\mathrm{SiC}_{3}$
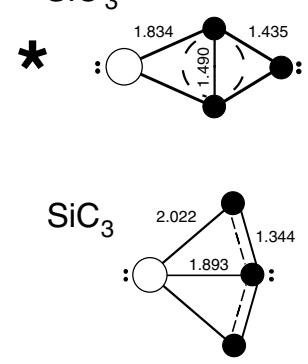

$\mathrm{SiC}_{3}$

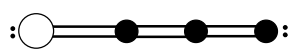

$\mathrm{SiC}_{5}$

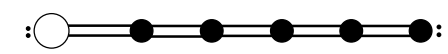

$\mathrm{SiC}_{6}$

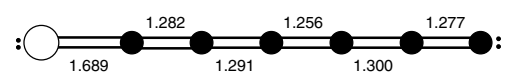

$\mathrm{SiC}_{7}$

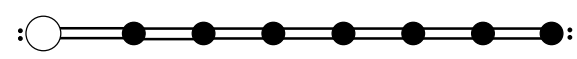

$\mathrm{SiC}_{8}$

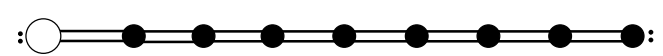

$\mathrm{SiC}_{2} \mathrm{H}$

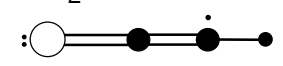

$\mathrm{SiC}_{4} \mathrm{H}$

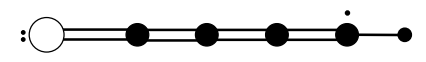

$\mathrm{SiC}_{5} \mathrm{H}$

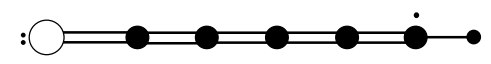

$\mathrm{SiC}_{6} \mathrm{H}$

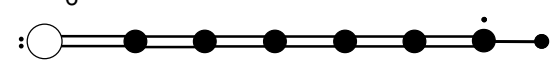

$\mathrm{SiCN}$

$x$

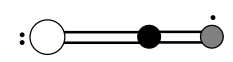

SiNC

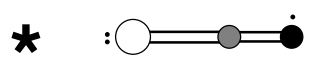

$\mathrm{HSiCN}$

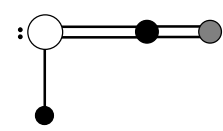

HSiNC
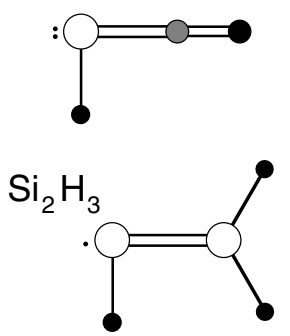

$\mathrm{H}_{2} \mathrm{CCSi}$

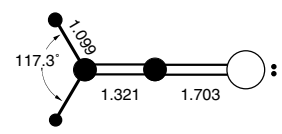

$\mathrm{H}_{2} \mathrm{CCCSi}$

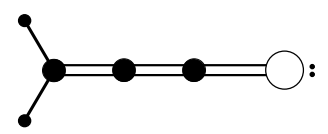

$\mathrm{H}_{2}$ CCCCSi

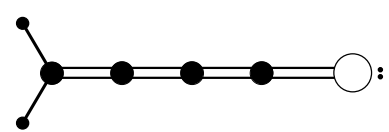

$\mathrm{SiC}_{2} \mathrm{~S}$

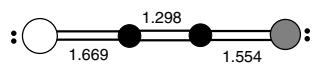

$\mathrm{SiC}_{3} \mathrm{~S}$

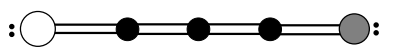

$\mathrm{SiC}_{4} \mathrm{~S}$

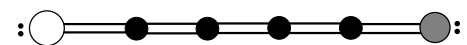

$\mathrm{SiC}_{6} \mathrm{~S}$

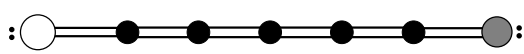

Figure 2. Silicon bearing molecules detected in this laboratory. With the exception of $\mathrm{SiC}$ and $\mathrm{SiC}_{2}$, all were detected in the past three years. Asterisks indicate those molecules detected in space as a result of this work. The convention described in figure 1 is used to denote the composition of each molecule. Bond lengths and angles were derived from isotopic measurements.

determined by the small width of the individual Doppler peaks.

Progress in detecting new silicon molecules in this laboratory has also benefited from instrumental improvements that have been made during the past several years. Of particular importance to the present work is the capability to survey large ranges in frequency under computer control. The spectrometer is now automated to the point that rotational spectra may be acquired rapidly either in a search mode, where individual spectra each limited in spectral range by the confocal cavity to about $0.5 \mathrm{MHz}$ are stitched together, or in a single-scan mode, where individual spectra are recorded at many different preselected frequencies. Continuous operation over many tens of hours or even days is now routine. A second crucial factor is sensitivity, which has been increased significantly by liquid nitrogen cooling a very low noise cryogenic microwave receiver and large confocal Fabry-Perot cavity [31].

One of the first silicon molecules detected with the Fourier transform microwave spectrometer is the rhom- 


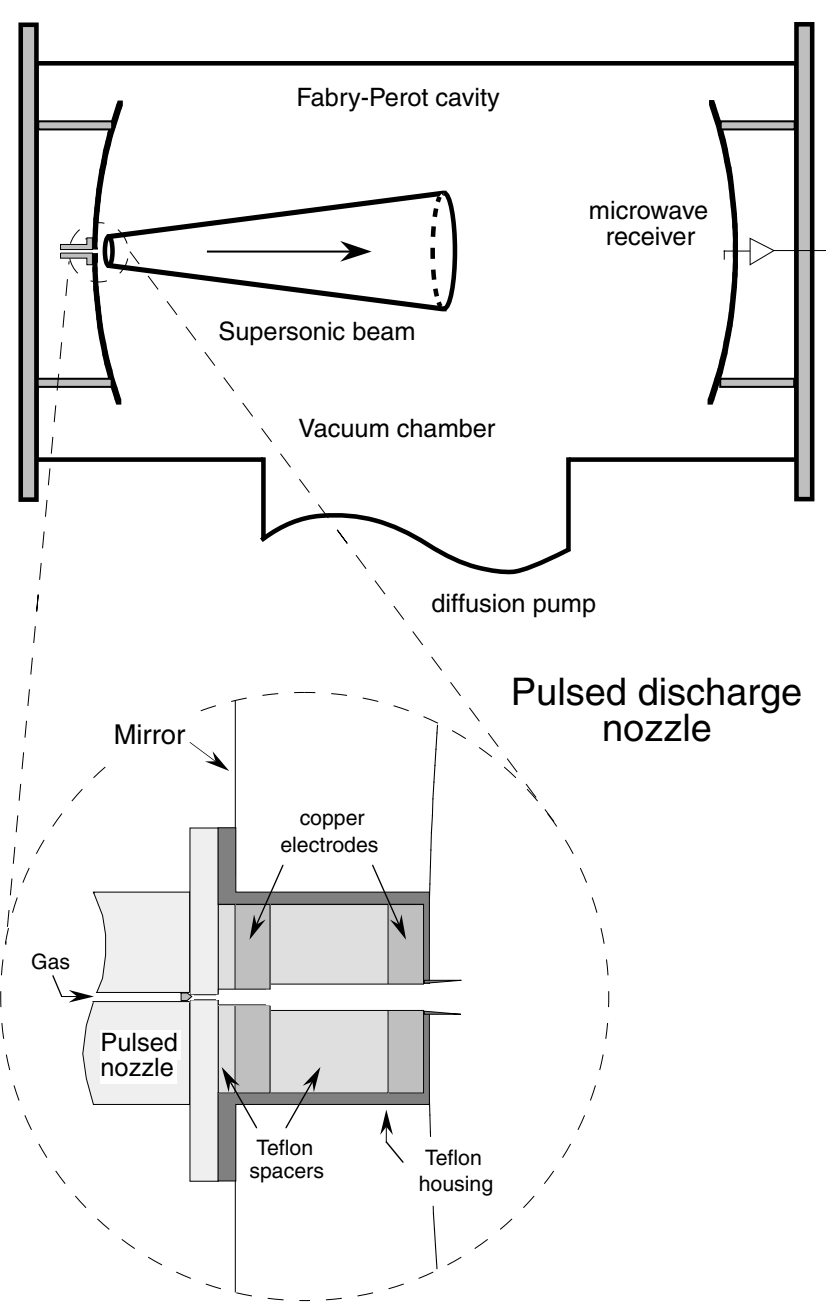

Figure 3. Schematic diagram of the FTM spectrometer showing the Fabry-Perot cavity, the axially oriented molecular beam, and the pulsed discharge source. The stagnation pressure of the gas behind the valve is $1-3 \mathrm{~atm}$, the discharge potential is $1000-1800 \mathrm{~V}$, and the length of the gas pulse is typically $200-350 \mu$ s. The diametre of the opening orifice of the nozzle immediately following the valve is $1 \mathrm{~mm}$. A pulsed valve working at $6-10 \mathrm{~Hz}$, open less than $1 \%$ of the time, is required to keep the pressure in the chamber low enough to form a good molecular beam.

boidal ring $\mathrm{SiC}_{3}$ (figure 1), a long postulated but previously unknown molecule with a remarkable internal or 'transannular' carbon-carbon bond. Once we had good rest frequencies in hand, we quickly detected seven lines at precisely the right frequencies in the circumstellar shell of IRC +10216 [32], one of the best astronomical sources of complex molecules. Rhomboidal $\mathrm{SiC}_{3}$ is the sixth and largest reactive ring molecule that has been conclusively identified in space.

Rotational transitions of rhomboidal $\mathrm{SiC}_{3}$ and its rare isotopic species were first observed in the centimetre- wave band [33] in a pulsed discharge through a dilute mixture of diacetylene and silane. At most four rotational transitions of each isotopic species lie within the frequency range of our FTM spectrometer, but this number alone is sufficient to establish unambiguously the existence of this exotic ring, and to allow a precise determination of its geometry. If $\mathrm{SiC}_{3}$ were strictly planar and rigid, two parameters would suffice to determine the entire spectrum (the three moments of inertia constrained by the Pythagorean theorem), but to describe the actual deformable molecule to the accuracy needed to conduct a radio astronomical search requires the three rotational constants and several centrifugal distortion constants. Using our free-space absorption spectrometer, the millimetre-wave spectrum of $\mathrm{SiC}_{3}$ was measured [34] to within a few parts in $10^{7}$ up to $300 \mathrm{GHz}$, and the nine leading centrifugal distortion constants were determined to fairly high precision as well.

A total of 11 millimetre-wave rotational transitions of $\mathrm{SiC}_{3}$ have now been detected in IRC+10216 with the $12 \mathrm{~m}$ NRAO telescope on Kitt Peak. The lines are fairly weak $(\sim 5 \mathrm{mK})$, but all have a mean radial velocity of $-26(3) \mathrm{km} \mathrm{s}^{-1}$, precisely that found for most other molecules in this source. The rotational excitation of $\mathrm{SiC}_{3}$ is similar to that of $\mathrm{SiCC}$, with a low rotational temperature within the $K$-stacks of $10-20 \mathrm{~K}$, and a high rotational temperature across them of roughly $50 \mathrm{~K}$, which probably is fairly close to the kinetic temperature of the ambient $\mathrm{H}_{2}$ gas, the main constituent of the shell. The column density of $\mathrm{SiC}_{3}$ is calculated to be $4.3 \times 10^{12} \mathrm{~cm}^{-2}$ assuming a shell diametre of $60^{\prime \prime}$, perhaps $50 \%$ larger than that of $\mathrm{SiC}_{4}$.

Two other silicon molecules that were detected recently in the laboratory and then in space with radio telescopes are the isovalent radicals $\mathrm{SiCN}$ and $\mathrm{SiNC}$. Intense rotational FTM lines of both species were observed first at centimetre-wavelengths in a discharge through silane and cyanoacetylene, and then at millimetre-wavelengths with our free-space spectrometer [35]. Like $\mathrm{SiC}_{3}$, determination of the leading centrifugal distortion constants is required for an astronomical search because the most intense radio lines of these radicals are expected in a warm circumstellar source to lie in the $80-120 \mathrm{GHz}$ frequency range, where centrifugal distortion is important.

$\mathrm{SiCN}$ was discovered first in IRC+10216 [36], followed very recently by SiNC [37], using the powerful IRAM $30 \mathrm{~m}$ radio telescope. Three rotational transitions of $\mathrm{SiCN}$, each split by lambda-doubling, were detected at the $5 \mathrm{mK}$ level; from the characteristic cusp shapes of the line profiles, it appears that $\mathrm{SiCN}$, like many other radicals, is largely confined to the outer molecular envelope that surrounds the central star. The column 
density is estimated to be $2 \times 10^{12} \mathrm{~cm}^{-2}$, which is about 20 times smaller than that of $\mathrm{SiC}$ and $\mathrm{MgNC}$, but comparable to that of $\mathrm{MgCN}$. The strength of the SiNC lines and the derived column density of SiNC are similar to that of $\mathrm{SiCN}$, as one might expect from the comparable polarity and stability of these two nearly isoenergetic radicals ( $\mathrm{SiNC}$ is calculated to lie only $5 \mathrm{kcal} \mathrm{mol}^{-1}$ higher in energy). The formation mechanism of $\mathrm{SiCN}$ and $\mathrm{SiNC}$ is not well understood, but both may be formed via ion-molecule reactions, neutral-neutral reactions, or reactions on the interstellar dust grains.

Other silicon molecules of astronomical interest are (i) two low-lying rearrangements of $\mathrm{SiC}_{3}$ : a triplet chain and a second rhomboidal isomer [38], (ii) the recently discovered chain $\mathrm{H}_{2} \mathrm{CCSi}$ [39], and (iii) isoelectronic $\mathrm{HSiCN}$ and $\mathrm{HSiNC}$ [40]. Although $\mathrm{H}_{2} \mathrm{CSi}$ has not been found in space [41], $\mathrm{H}_{2} \mathrm{CCSi}$ is a good candidate for astronomical discovery because it is much more polar $(\mu=1.21 \mathrm{D}$ versus $\mu \sim 0.3 \mathrm{D})$ and because isovalent $\mathrm{H}_{2} \mathrm{CCC}$ has been detected in both interstellar and circumstellar sources. $\mathrm{HSiCN}$ and $\mathrm{HSiNC}$ were detected in the same molecular beam discharge source that produces strong lines of SiCN and SiNC, and precise spectroscopic constants were derived for each from the centimetre-wave data. Although there is no evidence for either species in published astronomical surveys, HSiCN $(\mu=3.5 \mathrm{D}$ [42]) and $\operatorname{HSiNC}(\mu=2.4 \mathrm{D})$ are highly polar, so dedicated astronomical searches are warranted. $\mathrm{HSiCN}$ is isovalent to $\mathrm{HCCN}$, a well studied laboratory molecule that possesses a large amplitude $\mathrm{CCH}$ bending motion and a non-rigid structure [43]. A structural analysis is underway to determine whether $\mathrm{HSiCN}$, like $\mathrm{HCCN}$, also possesses large amplitude bending.

\section{Postulated silicon molecules of laboratory and astronomical interest}

Although many new reactive silicon molecules have been discovered recently, the radio spectra of many (some with only a few atoms) remain unknown. Small molecules that are strong candidates for laboratory and astronomical detection include the diatomic molecule $\mathrm{SiP}$ and the triatomic molecules $\mathrm{SiNN}$ and $\mathrm{SiCO}$ shown in figure 4. $\mathrm{SiN}, \mathrm{PN}$, and $\mathrm{CP}$ have all been detected in at least one astronomical source [36, 44, 45], but the lack of accurate spectroscopic data has precluded an astronomical search for SiP. Strong optical spectra of SiP were recently measured in the laboratory [46] using a similar production scheme to the one we are likely to employ, i.e., a discharge through silane and phosphine $\left(\mathrm{PH}_{3}\right)$.

Despite the availability of high level $a b$ initio calculations [47-49] and the identification of $\mathrm{SiNN}, \mathrm{SiCO}$, and $\mathrm{SiCS}$ in an inert matrix by electron spin resonance [50],

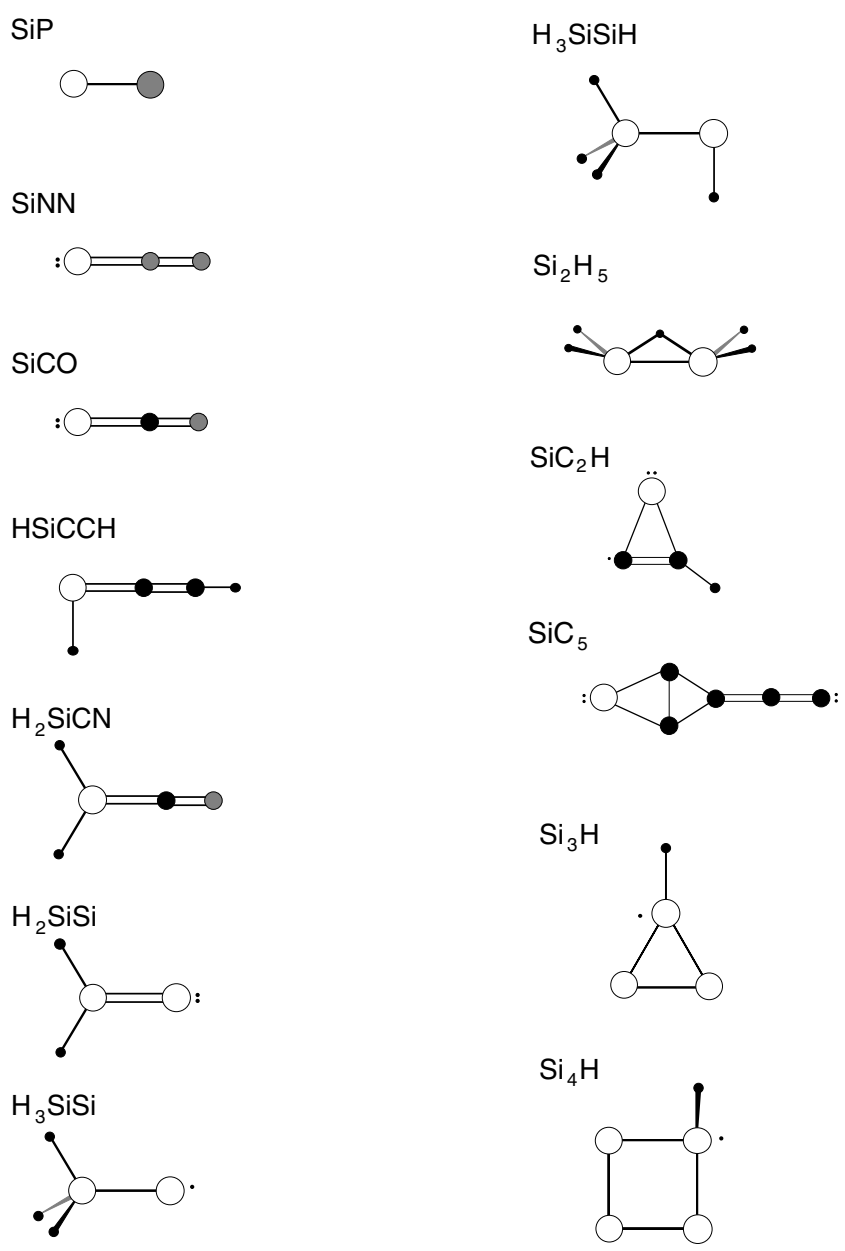

Figure 4. Postulated silicon molecules that are good candidates for laboratory and astronomical detection. The convention described in figure 1 is used to denote the composition of each molecule.

these simple molecules have eluded gas-phase detection, even though the isovalent triplet chains CCO and CCS have been the subjects of numerous experimental and astronomical investigations. The SiNN radical is potentially the most interesting of the three, because quantum chemical calculations suggested until recently that two distinct isomers, a tightly bound and a loosely bound structure, compete for the ground state; at the coupled cluster level of theory, however, the loose structure is not a minimum on the potential surface [51]. Experimental confirmation of this prediction is highly desirable, as is a precise determination of the $\mathrm{N}-\mathrm{N}$ bond length, because the theoretical $\mathrm{N}-\mathrm{N}$ distance is apparently sensitive to the level of theory and size of the basis set.

There is very little experimental or theoretical information on partially hydrogenated silicon molecules such as $\mathrm{H}_{2} \mathrm{SiCN}$, HSiCCH, $\mathrm{H}_{2} \mathrm{SiCCH}$, longer such chains, or the related isocyanides, even though these species are 
closely related in structure and composition to known astronomical molecules. Most of these chains possess linear or nearly linear heavy atom backbones, and as a result rotational spectra of nearly symmetric prolate tops with nearly harmonically related transitions that are recognized readily against the background of unrelated lines present in discharge sources such as ours. In addition to their astronomical interest, systematic studies of analogous systems such as $\mathrm{SiH}_{n} \mathrm{CCH}$ $(n=0-3)$ and $\mathrm{SiH}_{n} \mathrm{CN}(n=0-3)$ might significantly improve our understanding of the production mechanisms at work in our discharge source.

The astronomical detection of $\mathrm{SiC}_{3}$ implies that larger silicon rings exist at detectable abundances in space, and will be discovered there once precise laboratory frequencies are in hand. There are few high level theoretical calculations for systems larger than $\mathrm{SiC}_{3}$ to guide laboratory searches, but many low lying polar isomers of these larger silicon molecules are expected because of the tendency of silicon to prefer single rather than double or triple bonds, and to form both cyclic and linear isomers fairly readily. For example, Gomei et al. [52] and Zdetsis et al. [53] concluded on the basis of $a b$ initio calculations that silicon carbides up to $\mathrm{SiC}_{6}$ possess several low lying ring or ring-chain isomers. For $\mathrm{SiC}_{6}$, high level calculations [53] predicted a ring isomer with $\mathrm{C}_{2 \mathrm{v}}$ symmetry only $5 \mathrm{kcal} \mathrm{mol}^{-1}$ higher in energy than the linear chain that we have already observed with high signal-to-noise.

Many silicon carbide molecules may possess strong electronic transitions of astronomical interest; $\mathrm{SiC}$, for example, has strong such transitions [21, 22] and, as mentioned, optical bands of $\mathrm{SiC}_{2}$ have long been observed in the atmospheres of cool stars [24]. At the densities at which chains up to $\mathrm{SiC}_{7}$ are produced in our molecular beam, such spectra may be detectable by current laser techniques such as laser-induced fluorescence or cavity ring down spectroscopy. Compared with the isoelectronic pure carbon chains, these chains (or rings) have the advantage that their production may now be monitored and optimized in the radio band as described here. If electronic transitions are detected, it may be possible to confirm the identifications by means of microwave-optical double resonance [54].

Silicon hydrides of the form $\mathrm{Si}_{2} \mathrm{H}_{m}$ with $m>2$ are also excellent candidates for laboratory detection because we already have evidence that $\mathrm{Si}_{2} \mathrm{H}_{2}$ [55], $\mathrm{H}_{2} \mathrm{Si}_{2} \mathrm{H}$, and other silicon hydrides are readily produced in a discharge through silane. Laboratory detection of $\mathrm{H}_{3} \mathrm{SiSi}$ (figure 4) is a good place to start because this molecule is calculated to be polar and nearly isoenergetic to $\mathrm{H}_{2} \mathrm{SiSiH}[56,57]$, and to have the easily recognizable rotational spectrum of a nearly symmetric prolate top. Polar isomers of $\mathrm{Si}_{2} \mathrm{H}_{4}$, including $\mathrm{H}_{3} \mathrm{SiSiH}$, are good candidates for laboratory detection for the same reasons.

Another class of silicon molecules that is of interest are silicon hydrides with three or more silicon atoms. Detection of longer chains and larger ring radicals (e.g., $\mathrm{Si}_{3} \mathrm{H}_{2}, \mathrm{Si}_{3} \mathrm{H}, \mathrm{Si}_{4} \mathrm{H}$ ) should be possible because the time-of-flight mass spectrum of a discharge of silane measured in our laboratory indicates that $\mathrm{Si}_{3}$ and larger silicon clusters are only about $10 \%$ less abundant than the ubiquitous $\mathrm{Si}_{2}$ dimer, and because the photodetachment spectrum of some of the proposed molecules has been measured by Neumark and coworkers [58]. Many chain and ring isomers of comparable stability probably exist for silicon hydrides with three or more silicon atoms because of the ability of silicon to be either singly or multiply bound to adjacent silicon atoms in the cluster. Direct spectroscopic observations of larger silicon hydrides should provide us with a better understanding of silicon cluster structure, and how such clusters form in the laboratory and in space. Such studies may also provide an important challenge for computational techniques, because of the subtle and ambiguous character of silicon bonding.

Once rotational lines of the normal isotopic species have been found, a number of interesting follow-up investigations are worth pursuing, including (i) detection of rare isotopic spectra and determinations of molecular structure, (ii) detection of rotational satellites from low lying vibrational states and, where appropriate, (iii) millimetre-wave measurements to determine the leading centrifugal distortion constants of molecules whose strongest radio lines fall in the millimetre-wave band. Such studies provide useful information for subsequent experiments at infrared or optical wavelengths, and possible insights into a wide range of important phenomena in chemical physics, including molecular structure and rigidity, chemical bonding, and intramolecular vibrational relaxation.

We are indebted to E. S. Palmer for technical assistance in the construction and maintenance of the FTM spectrometer, and E. W. Gottlieb for assistance with software for spectral acquisition and analysis. We also thank those colleagues who contributed to some of the original papers referred to here: A. J. Apponi, M. E. Sanz, R. Mollaaghababa, J. M. Vrtilek and M. Guélin.

\section{Note added in proof}

Very recently three new silicon molecules have been detected in our laboratory: $\mathrm{Si}_{3}$ and a low-lying monobridged isomer of $\mathrm{Si}_{2} \mathrm{H}_{4}$ have been observed by FTM spectroscopy, and $\mathrm{Si}_{3} \mathrm{C}$ has been observed by resonant two-colour, two-photon ionization spectroscopy. 


\section{References}

[1] Jenkins, E. B., 1987, Interstellar Processes, edited by D. J. Hollenbach, and H. A. Thronson (Boston: D. Reidel) p. 533.

[2] Wilson, R. W., Penzias, A. A., Jefferts, K. B., Kutner, M., and Thaddeus, P., 1971, Astrophys. J. Lett., 167, L97.

[3] Snyder, L. E., and Buhl, D., 1973, Astrophys. J. Lett., 189, L31.

[4] Buhl, D., Snyder, L. E., Lovas, F. J., and Johnson, D. R., 1974, Astrophys. J. Lett., 192, L97.

[5] Alcolea, J., Bujarrabal, V., and Gallego, J. D., 1989, Astron. Astrophys., 211, 187.

[6] Törring, T., 1968, Z. Naturforsch., 23a, 777.

[7] Raymonda, J. W., Muenter, J. S., and Klemperer, W., 1968, J. chem. Phys., 52, 3458.

[8] Manson Jr., E. L., Clark, W. W., Delucia, F. C., and Gordy, W., 1977, Phys. Rev. A, 15, 223.

[9] Hoeft, J., 1965, Z. Naturforsch., 20a, 1327.

[10] Davis, J. H., Blair, G. N., Van Till, H., and Thaddeus, P., 1974, Astrophys. J. Lett., 190, L117.

[11] Elitzur, M., 1992, Ann. Rev. Astron. Astrophys., 30, 75.

[12] Olofsson, H., Lindevist, M., Nyman, L-Å., and Winnberg, A., 1998, Astron. Astrophys., 329, 1059.

[13] Morris, M., Gilmore, W., Palmer, P., Turner, B. E., and Zuckerman, B., 1975, Astrophys. J. Lett., 199, L47.

[14] Cernicharo, J., Guélin, M., and Kahane, C., 2000, Astron. Astrophys. Suppl., 142, 181.

[15] Bieging, J. H., and NguYen-Quang-Rieu, 1989, Astrophys. J. Lett., 343, L25.

[16] Glasssgold, A. E., and Mamon, G. A., 1992, Chemistry and Spectroscopy of Interstellar Molecules, edited by D. K. Bohme, E. Herbst, N. Kaifu, and S. Saito (Tokyo: University of Tokyo Press) p. 261.

[17] McCabe, E. M., 1982, Mon. Not. R. astron. Soc., 200, 71.

[18] Cernicharo, J., Gottlieb, C. A., Guélin, M., Thaddeus, P., and Vrtilek, J. M., 1989, Astrophys. J. Lett., 341, L25.

[19] Bernath, P. F., Rogers, S. A., O'Brien, L. C., Brazier, C. R., and Mclean, A. D., 1988, Phys. Rev. Lett., 60, 197.

[20] Brazier, C. R., O’Brien, L. C., and Bernath, P. F., 1989, J. chem. Phys., 91, 7384.

[21] Ebben, M., Drabbels, M., and ter Meulen, J. J., 1991, Chem. Phys. Lett., 176, 404.

[22] Butenhoff, T. J., and Rohlfing, E. A., 1991, J. chem. Phys., 95, 3939.

[23] Mollahghababa, R., Gottlieb, C. A., Vrtilek, J. M., and Thaddeus, P., 1990, Astrophys. J. Lett., 352, L21.

[24] Merrill, P. W., 1926, Publ. astron. Soc. Pacif., 38, 175; Sanford, R. F., Publ. astron. Soc. Pacif., 38, 177.

[25] Kleman, B., 1956, Astrophys. J., 123, 162.

[26] Michalopoulos, D. L., Geusic, M. E., LangridgeSmith, P. R. R., and Smalley, R. E., 1984, J. chem. Phys., 80, 3556.

[27] Thaddeus, P., Cummins, S. E., and Linke, R. A., 1984, Astrophys. J. Lett., 283, L45.

[28] Gottlieb, C. A., Vrtilek, J. M., and Thaddeus, P., 1989, Astrophys. J. Lett., 343, L29.

[29] Nielsen, I. B. D., Allen, W. D., Csaszar, A. G., and Schaefer III, H. F., 1997, J. chem. Phys., 107, 1195.

[30] Ohishi, M., Kaifu, N., Kawaguchi, K., Murakami, A., Saito, S., Yamamoto, S., Ishikawa, S. I., Fujita,
Y., Shiratori, Y., and Irvine, W. M., 1989, Astrophys. J. Lett., 345, L83.

[31] McCarthy, M. C., Chen, W., Travers, M. J., and Thaddeus, P., 2000, Astrophys. J. Suppl., 129, 611.

[32] Apponi, A. J., McCarthy, M. C., Gottlieb, C. A., and Thaddeus, P., 1999, Astrophys. J. Lett., 516, L103.

[33] McCarthy, M. C., Apponi, A. J., and Thaddeus, P., 1999, J. chem. Phys., 110, 10645.

[34] Apponi, A. J., McCarthy, M. C., Gottlieb, C. A., and Thaddeus, P., 1999, J. chem. Phys., 111, 3911.

[35] Apponi, A. J., McCarthy, M. C., Gottlieb, C. A., and Thaddeus, P., 2000, Astrophys. J. Lett., 536, L55.

[36] Gú́lin, M., Muller, S., Cernicharo, J., Apponi, A. J., MCCarthy, M. C., Gottlieb, C. A., and Thaddeus, P., 2000, Astron. Astrophys., 363, L9.

[37] GuÉlin, M. et al., unpublished.

[38] McCarthy, M. C., Apponi, A. J., and Thaddeus, P., 1999, J. chem. Phys., 111, 7175; McCarthy, M. C., Apponi, A. J., Gottlieb, C. A., and Thaddeus, P., 2000, Astrophys. J., 538, 766.

[39] McCarthy, M. C., and Thaddeus, P., 2002, J. molec. Spectrosc., 211, 228.

[40] Sanz, M. E., McCarthy, M. C., and Thaddeus, P., 2002, Astrophys. J. Lett., 577, L71.

[41] Izuha, M., Yамамото, S., and Saito, S., 1994, Can. J. Phys., 72, 1206.

[42] Sanz, M. E., McCarthy, M. C., and Thaddeus, P., 2002 , in preparation.

[43] Hung, P. Y., Sun, F., Hunt, N. T., Burns, L. A., and Curl, R. F., 2001, J. chem. Phys., 115, 9331, and references therein.

[44] Guélin, M., Cernicharo, J., Paubert, G., and Turner, B. E., 1990, Astron. Astrophys., 230, L9.

[45] Turner, B. E., 1992, Astrophys. J. Lett., 388, L35.

[46] Jakubek, Z., Nakhate, S. G., and Simard, B., 2002, J. chem. Phys., 116, 6513.

[47] DeKock, R. L., Grev, R. S., and Schaefer III, H. F., 1988, J. chem. Phys., 89, 3016.

[48] Suter, H. U., Huang, M.-B., and Engels, B., 1994, J. chem. Phys., 101, 7686.

[49] Petraco, N. D., Brown, S. T., Yamaguchi, Y., and Schaefer III, H. F., 2000, J. chem. Phys., 112, 3201.

[50] Lembke, R. R., Ferrante, R. F., and Weltner Jr., W., 1977, J. Amer. chem. Soc., 99, 416.

[51] Ignatyev, I. S., and Schaefer III, H. F., 1992, J. phys. Chem., 96, 7632.

[52] Gomei, M., Kishi, R., Nakajima, A., Iwata, S., and Kaya, K., 1997, J. chem. Phys., 107, 10051.

[53] Zdetsis, A., Engels, B., Hanrath, M., and Peyerimhoff, S. D., 1999, Chem. Phys. Lett., 302, 288.

[54] Nakajima, M., Sumiyoshi, Y., and Endo, Y., 2002, Rev. sci. Instrum., 73, 165.

[55] Cordonnier, M., Bogey, M., Demuynck, C., and Destombes, J.-L., 1992, J. chem. Phys., 97, 7984, and references therein.

[56] Curtiss, L. A., Raghavachari, K., Deutsch, P. W., and Pople, J. A., 1991, J. chem. Phys., 95, 2433.

[57] PaK, C., Rienstra-Kiracofe, J., and Schaefer III, H. F., 2000, J. phys. Chem. A, 104, 11232.

[58] Xu, C., Taylor, T. R., Burton, G. R., and Neumark, D. M., 1998, J. chem. Phys., 108, 7645.

[59] Turner, B. E., 1995, Astrophys. Space Sci., 224, 297. 